
\title{
R Research S Surare \\ Is Virtual Clinic the Way Forward: Patient Satisfaction Comparing Phone Clinic vs. Conventional Clinic
}

Benjamin Wall ( $\nabla$ wall.ben123@gmail.com )

FSH: Fiona Stanley Hospital https://orcid.org/0000-0003-1590-366X

\section{Patrick Daly}

Rockingham General Hospital

Aidan Dunnill

Rockingham General Hospital

Jessica Osan

Rockingham General Hospital

Kit Brogan

Rockingham General Hospital

Research article

Keywords: virtual clinic, Patient satisfaction, phone clinic vs. conventional clinic, Outpatient clinics

Posted Date: February 11th, 2021

DOI: https://doi.org/10.21203/rs.3.rs-193370/v1

License: (c) (1) This work is licensed under a Creative Commons Attribution 4.0 International License. Read Full License 


\section{Abstract}

Background:

Outpatient clinics play a vital role in assessing and treating patients. They traditionally involve face-toface consultation with a clinician to diagnose and treat many medical and surgical conditions. During the COVID-19 pandemic many of these traditional methods were replaced with telemedicine to minimise patient interaction and therefore potential exposure. This study utilises data from patient questionnaires with the aim of determining patient satisfaction in regard to telemedicine.

Method:

A list of patients who attended the Rockingham General hospital outpatient Orthopaedic phone clinic was obtained between $23^{\text {rd }}$ of March and $30^{\text {th }}$ of May, this list was then filtered into a randomised generator to provide a call list of patients. Patients were contacted and verbal consent was obtained to participate in satisfaction questionnaire that was completed via phone.

Results:

A total of 100 patients completed the questionnaire - 50 from elective surgery clinics and 50 from fracture clinics. The mean time for participants' one-way commute to the hospital was 21 minutes ( 2 to 60 minutes). Only $4 \%$ of telehealth consultations resulted in patients being forced to miss work or school. $13 \%$ of patients were required to attend the hospital on the day of their consultation for either radiological imaging or for application or removal of casts or splint. We observed consistently high satisfaction scores across all parameters, in both elective and fracture clinics, culminating in a mean score of 9.08/10 on the Likert scale. In the qualitative component of the questionnaire, patients frequently acknowledged a high quality of care despite adverse circumstances.

Conclusion:

Telemedicine continues to develop and become a more widely accessible tool in the provision of outpatient orthopaedic care. With its ever-increasing availability and acceptance, it should play a more central role in delivering effective and efficient healthcare to all patients.

\section{Introduction}

Outpatient clinics play a vital role in assessing and treating patients. These clinics have traditionally involved face-to-face consultations with a clinician to diagnose and treat a wide range of medical and surgical conditions.

Telemedicine, defined as the remote diagnosis and treatment of patients through telecommunications technology, has served as an adjunct to conventional healthcare provision since its earliest published utilisation in the 1970's.(1) Despite tremendous advances in the accessibility of mobile phones and 
wireless internet over the last 20 years, the application of this service has not yet been universally adopted in the medical setting, in part due to the perception that it is less effective than traditional face to face consultations.(2)

The unanticipated advent of the COVID-19 pandemic precipitated significant acceleration of the adoption of telemedicine. To sequester healthcare resources, as well as to limit the exposure of patients and healthcare professionals to the virus, hospital systems worldwide saw the cancellation of elective surgeries and the restriction of hospital visits.(3) While the rapid adaptation of these systems has proved challenging, we must also consider these adverse circumstances as an opportunity to embrace the technology at our disposal, and in doing so, make significant advances in the quality and efficiency of healthcare services.

The Department of Orthopaedic Surgery at Rockingham General Hospital sees approximately 1400 outpatient consultations each month, with $97 \%$ of these consultations face-to-face in nature. From March 23rd 2020, in keeping with the recommendations of the Department of Health of Western Australia, elective outpatient clinics were converted into an entirely telemedicine based service, and subsequent face-to-face appointments could be booked only if absolutely clinically indicated.(3)

Telemedicine poses many potential opportunities for the improvement of patient care, including increased availability of specialised healthcare to underserved geographically remote populations.(4-7) Furthermore, in a climate where clinical demands often outweigh available resources, telemedicine has been shown to be cost-effective, while also increasing the overall efficiency of healthcare systems. $(4,8,9)$ Although studies have revealed telemedicine to be a safe and robust pathway in the management of specific orthopaedic presentations, it is important to maintain patient satisfaction as a key indicator of healthcare quality, regardless of its delivery modality $(4,9,10)$.

The primary aim of this pilot study was to assess patient satisfaction outcomes with the provision of a predominantly telemedicine-based orthopaedic service. Such outcomes will assist in guiding us in the establishment of a patient-centred care pathway that reflects the vast technological advances of recent times.

\section{Method}

Due to the outbreak of COVID-19 and stringent precautions put in place, patients were required to attend outpatient clinics via telephone between the dates $30 / 03 / 2020$ and the 30/05/2020. All patients were notified the week prior to their appointment by text message or phone call to inform them of the changes with a subsequent confirmation text message sent the day prior to their appointment. Patients were then called at their allocated appointment time by a member of the orthopaedic team from resident to consultant grade.

To assess patient satisfaction, a questionnaire was designed by doctors from the orthopaedic department at Rockingham General hospital, based on previous satisfaction questionnaires utilised 
(Australian Commission on safety and quality in health care).(6) All questions were reviewed in a group discussion involving several orthopaedic surgeons, nursing staff and allied health staff within the hospital. The questionnaire was divided into two parts: part 1 pertained to demographic information for each participating patient, part 2 addressed the response of each patient to the telephone consult.

The questionnaire was used on a retrospective convenience sample of patients who had received a phone consult over the COVID-19 period. As this was a pilot study a sample size calculation was not performed. The target was 100 completed surveys which was felt to be an obtainable number and sufficient to gauge the initial response. A list of patients seen via phone clinic was acquired from outpatient department electronic records ordered by date of appointment. The patient order was then randomised using online generator. Patients were contacted in the order listed after randomisation. Patients were excluded if they were seen face to face or had a diagnosis of dementia or cognitive impairment recorded in their medical record. Patients were contacted once via phone, patients who did not answer were not included in the study.

Patients were contacted by telephone and consented to participate in the research, verbal telephone consent was obtained at the commencement of each call. The nature of the study was described to each patient and they had the option of refusing to be questioned and it was explained that this would have no impact on the future care they would receive. For those patients below the age of consent the data was retrieved from the next of kin (NOK) who had attended the phone consult on the patient's behalf.

Data collection was performed by four individual reviewers who were not involved in the care provided to the patients. To reduce bias patients were blinded to specific researcher occupation, who identified as doctors from the hospital at which this research was taking place.

Demographic information was obtained from the hospital episode statistics (HES) data following discussion and ethical approval from the audit department. Data regarding the patient's response to the clinic was obtained through use of a Likert scale with responses ranging 1-10 ( $1=$ not at all satisfied, $10=$ completely satisfied. All patients were also given the opportunity to provide qualitative data, being invited to provide any additional comments on their telephone clinic experience following their completion of the questionnaire.

\section{Results}

\section{Patient demographics}

100 patients completed the satisfaction questionnaire - 50 from elective surgery clinics and 50 from fracture clinics. Of the 50 elective surgery consultations, 44 were pre-operative and 6 were post-operative. Elective orthopaedic consultations dealt with a wide range of orthopaedic presentations - 15 shoulder, 14 knee, 9 hip, 2 elbow, 2 foot and ankle and 8 other consultations were included in the survey. Of the fracture clinic consultations, 47 were pre-operative and 3 were post-operative (Table 1). The mean age of 
study participants was 48.7 years ( 7 - 85 ). $41 \%$ of participants were male, while $59 \%$ were female (Table 2).

\section{Patient convenience}

The mean time for participants' one-way commute to the hospital from their home was 21 minutes ( 2 to 60 minutes) (Table 3). Only 4\% of telehealth consultations resulted in patients being forced to miss work or school. $13 \%$ of patients were required to attend the hospital on the day of their consultation for either radiological imaging or for application or removal of casts or splints.

\section{Patient satisfaction}

Overall satisfaction with the Telemedicine consultations was high with a mean score of 9.00/10 (9.22 in the Elective Clinics, 8.78 in Fracture Clinics). (Table 4) Highest mean satisfaction scores recorded were in relation to the clearness of instruction sent to patients prior to their appointment $(9.18 / 10)$ and understanding the recommendations of the doctor following their appointment (9.18/10). Lowest mean satisfaction scores were in relation to convenience of the Telemedicine consultation (8.96/10) and waiting time on the day of the appointment $(8.96 / 10)$.

\section{Appointment outcome}

Regarding outcomes of the telemedicine appointments, $38 \%$ of patients were discharged from the orthopaedic service, $39 \%$ required further follow-up appointments, while $23 \%$ did not respond to this part of the questionnaire. Based on their experience of the Telemedicine consultation, $53 \%$ of patients expressed a preference for face-to-face consultations for future appointments, while $47 \%$ would prefer a Telemedicine consultation.

\section{Qualitative Responses}

Common themes among positive responses included less anxiety related to acquiring a COVID-19 infection, the convenience of not having to drive (particularly in those unable to drive due to injury), feeling less rushed during the consultation and the courteous manner of the doctors. Frequent negative responses included difficulty establishing rapport with the doctor (particularly in first-time consultations), disappointment in a lack of definitive diagnosis without physical examination, and frustration with the Telemedicine appointment not occurring at the exact time assigned. (Table 5)

\section{Discussion}

We observed consistently high satisfaction scores across all parameters, in both elective and fracture clinics, culminating in a mean score of $9.08 / 10$ on the Likert scale. In the qualitative component of the questionnaire, patients frequently acknowledged a high quality of care despite adverse circumstances. 
Telemedicine satisfaction rates are comparable to those of conventional orthopaedic outpatient visits.(6, 11-15) Patients who experience telemedicine consultations are more likely to opt for this mode of orthopaedic care in the future.(6, 7) A study by Buvik et al.(2019) performed a randomised control trial comparing in person or videoconference review.(6) Of the 389 patients $99 \%$ of patients rated their consultation satisfactory or very satisfactory, regardless of how they were seen.(6) In addition, $86 \%$ of patients assigned to the videoconference arm expressed a preference for telemedicine for future consultations. Similarly, a non-randomised study in Pennsylvania in 2018 found comparable satisfaction rates between face-to-face and telemedicine visits, while only $8 \%$ of telemedicine patients requested inperson care for their next visit.(14)

The majority of these studies took place at tertiary orthopaedic centres in Europe, with patients teleconferencing from a regional facility, closer to their homes. An economic evaluation performed on the Norwegian randomised control trial found patients were required to travel a mean one-way distance of $248 \mathrm{~km}$, with a mean one-way travel time of 4 hours and 37 minutes, in order to attend face-to-face consultations. $(5,12)$ In contrast, Rockingham General Hospital is a regional orthopaedic centre, with patients in this study having a mean one-way travel time of only 21 minutes. In addition, $13 \%$ of patients were required to attend the hospital on the day of their telemedicine. Cost-effectiveness for both healthcare providers and patients, including reduced travel costs, is frequently described in the literature as a major benefit of telemedicine. Due to the reduced distances compared with other studies this may explain why only $47 \%$ of patients in our study expressed a preference for telemedicine for future visits despite achieving very high satisfaction rates.(14)

Lack of technology literacy and access, as well as high implementation and maintenance costs are cited as barriers to telemedicine. $(16,17)$ Consultation delays due to technical difficulties can also significantly reduce the efficiency and patient satisfaction associated with the modality. $(6,15)$ Due to the need for a rapid implementation of remote consultations in our study, as well as a lack of internet and videoconferencing facilities at the hospital, all telemedicine visits were carried out over phone. However, our results show that patients found this modality to be convenient and accessible. Patients were not required to attend a remote site for video-conferencing facilities, were not faced with the frustration of technical delays and experienced minimal disruption of their daily activities. We recommend further costbenefit analyses be carried out into videoconferencing versus standard phone consultations.

In the qualitative component of the study, constructive feedback was encouraged to highlight aspects of the telemedicine service that warranted modification. Consequently, we would recommend assigning patients with a broader time range during which they would be called, as opposed to a specific appointment time. In other cases, patients disclosed frustration with an inability of the physician to arrive at a definite diagnosis due to a lack of physical examination during the consultation. Although this is certainly a major drawback to telemedicine as a healthcare modality, the phone consultation acted as a valuable tool in initiating a diagnostic and therapeutic care plan, whilst also minimising patients' risk of acquiring COVID-19 infection. 
While patient satisfaction was the primary focus of our study, a review of the pertinent literature reveals several additional benefits of telemedicine in the provision of orthopaedic care. Telemedicine poses a significant economic benefit with numerous financial analyses indicating the cost-effectiveness of the modality when compared with conventional visits. $(4,5,9,18-20)$ Virtual clinics are not only cost-efficient, but time-efficient for both patients and physicians. Travel and consultation times are decreased, while the utilisation of telemedicine as a triage tool reduces the number of emergency department referrals requiring a face-to-face consult.(21) Access to specialist care is consistently cited as a significant benefit of telemedicine, removing many of the geographical and logistical barriers that would otherwise prevent patients from availing of such services. $(6,7,20)$ In addition to the well-documented health benefits of decreased exposure to the hospital environment, telemedicine poses the theoretical benefit of reduced mobilisation on the often-compromised musculoskeletal system in orthopaedic patients. With virtual orthopaedic care in its relative infancy, it is difficult to conclude emphatically on the long-term accuracy of this style of care. However, early research reveals efficacy and accuracy levels comparable to those of face-to-face consults. $(6,7,15)$

\section{Limitations}

While the utilisation of telemedicine has multiple benefits, there are several limitations which have likely contributed towards its relatively low uptake figures prior to the COVID-19 pandemic. Physical examination remains an key component of the orthopaedic consultation.(22) Throughout our study, many patients revealed their frustrations at the inability to arrive at a conclusive diagnosis as is many incidences the diagnosis is dependent on the patients examination findings The lack of physical examination during this study also underlined the fact that virtual clinic visits may only be suitable for certain clinical presentations. Additional challenges to the widespread uptake of telemedicine by both physicians and patients include lack of awareness of the modality, poor levels of technological literacy and access, lack of perceived benefit and potential medicolegal exposure. $(16,22-24)$

The logical next step to progress our body of work is to perform an adequately powered single blinded comparative effectiveness, non-inferiority RCT to determine if telephone consults are as effective as face to face consultation without compromising patient care.

\section{Conclusion}

While the early results are promising, it is important to interpret them in the context of our study's limitations. Through this questionnaire, we have sought not to demonstrate statistical significance, nor drastically revolutionise the means in which orthopaedic care is delivered. Rather, this study offers a valuable snapshot of the patient's perspective of a rapid implementation of telemedicine services amid a global pandemic and provides useful information for the ongoing use of telehealth in appropriate patients. As such, the present study highlights the exciting potential of telemedicine in the future of orthopaedics, while simultaneously emphasising the value which patients place on human interaction. 


\section{Declarations}

\section{Ethics approval and consent to participate}

Not application- Quality improvement project

Consent for publication

Give full consent for publication

Availability of data and materials

Full data set is available for review

Competing interests

Not Applicable

\section{Funding}

Not Applicable

\section{Authors' contributions}

Benjamin Wall

Methodology, Writing-original draft, Writing-review \& editing

Patrick Daly

Validation, Writing-original draft, Writing-review \& editing

Aidan Dunnill

Methodology, Writing-review \& editing

Jess Osan

Investigation, Writing-review \& editing

Kit Brogan

Supervision, Writing-review \& editing

Acknowledgements

NA

\section{References}


1. Glazer E, Marshall C, Cunningham N. Remote pediatric consultation in the inner city: television or telephone? American journal of public health. 1978;68(11):1133-5.

2. Bashshur RL, Shannon G, Krupinski EA, Grigsby J. Sustaining and realizing the promise of telemedicine. Telemedicine and e-Health. 2013;19(5):339-45.

3. Government of Western Australia. Elective surgery to be scaled back to prepare for COVID-19 2020 [Available from: https://www.mediastatements.wa.gov.au/Pages/McGowan/2020/03/Electivesurgery-to-be-scaled-back-to-prepare-for-COVID-19.aspx.

4. Bellringer SF, Brogan K, Cassidy L, Gibbs J. Standardised virtual fracture clinic management of radiographically stable Weber $B$ ankle fractures is safe, cost effective and reproducible. Injury. 2017;48(7):1670-3.

5. Buvik A, Bergmo TS, Bugge E, Smaabrekke A, Wilsgaard T, Olsen JA. Cost-Effectiveness of Telemedicine in Remote Orthopedic Consultations: Randomized Controlled Trial. J Med Internet Res. 2019;21(2):e11330.

6. Buvik A, Bugge E, Knutsen G, Småbrekke A, Wilsgaard T. Patient reported outcomes with remote orthopaedic consultations by telemedicine: A randomised controlled trial. J Telemed Telecare. 2019;25(8):451-9.

7. Caffery LJ, Taylor M, North JB, Smith AC. Tele-orthopaedics: A snapshot of services in Australia. J Telemed Telecare. 2017;23(10):835-41.

8. Aponte-Tinao LA, Farfalli GL, Albergo JI, Plazzotta F, Sommer J, Luna D, et al. Face to Face Appointment vs. Telemedicine in First Time Appointment Orthopedic Oncology Patients: A Cost Analysis. Stud Health Technol Inform. 2019;264:512-5.

9. Brogan $\mathrm{K}$, Bellringer $\mathrm{S}$, Akehurst $\mathrm{H}$, Gee $\mathrm{C}$, Ibrahim N, Cassidy L, et al. Virtual fracture clinic management of fifth metatarsal, including Jones', fractures is safe and cost-effective. Injury. 2017;48(4):966-70.

10. Jayaram PR, Bhattacharyya R, Jenkins PJ, Anthony I, Rymaszewski LA. A new "virtual" patient pathway for the management of radial head and neck fractures. J Shoulder Elbow Surg. 2014;23(3):297-301.

11. Young $L$, Siden $H$, Tredwell S. Post-surgical telehealth support for children and family care-givers. J Telemed Telecare. 2007;13(1):15-9.

12. Harrison R, Macfarlane A, Murray E, Wallace P. Patients' perceptions of joint teleconsultations: a qualitative evaluation. Health Expect. 2006;9(1):81-90.

13. Kairy D, Tousignant M, Leclerc N, Côté AM, Levasseur M, Researchers TT. The patient's perspective of in-home telerehabilitation physiotherapy services following total knee arthroplasty. Int J Environ Res Public Health. 2013;10(9):3998-4011.

14. Sinha N, Cornell M, Wheatley B, Munley N, Seeley M. Looking Through a Different Lens: Patient Satisfaction With Telemedicine in Delivering Pediatric Fracture Care. J Am Acad Orthop Surg Glob Res Rev. 2019;3(9):e100. 
15. Gilbert AW, Jaggi A, May CR. What is the patient acceptability of real time 1:1 videoconferencing in an orthopaedics setting? A systematic review. Physiotherapy. 2018;104(2):178-86.

16. Ayatollahi H, Sarabi FZ, Langarizadeh M. Clinicians' Knowledge and Perception of Telemedicine Technology. Perspect Health Inf Manag. 2015;12(Fall):1C.

17. Burke BL, Jr., Hall RW. Telemedicine: Pediatric Applications. Pediatrics. 2015;136(1):e293-308.

18. Harno K, Arajärvi E, Paavola T, Carlson C, Viikinkoski P. Clinical effectiveness and cost analysis of patient referral by videoconferencing in orthopaedics. J Telemed Telecare. 2001;7(4):219-25.

19. Ohinmaa A, Vuolio S, Haukipuro K, Winblad I. A cost-minimization analysis of orthopaedic consultations using videoconferencing in comparison with conventional consulting. $\mathrm{J}$ Telemed Telecare. 2002;8(5):283-9.

20. Prada C, Izquierdo N, Traipe R, Figueroa C. Results of a New Telemedicine Strategy in Traumatology and Orthopedics. Telemed J E Health. 2020;26(5):665-70.

21. Mackenzie SP, Carter TH, Jefferies JG, Wilby JBJ, Hall P, Duckworth AD, et al. Discharged but not dissatisfied: outcomes and satisfaction of patients discharged from the Edinburgh Trauma Triage Clinic. Bone Joint J. 2018;100-b(7):959-65.

22. Makhni MC, Riew GJ, Sumathipala MG. Telemedicine in Orthopaedic Surgery: Challenges and Opportunities. J Bone Joint Surg Am. 2020;102(13):1109-15.

23. Kuszler PC. Telemedicine and integrated health care delivery: compounding malpractice liability. Am J Law Med. 1999;25(2-3):297-326.

24. Stanberry B. Legal and ethical aspects of telemedicine. J Telemed Telecare. 2006;12(4):166-75.

\section{Tables}


Table 1:

Descriptive statistics

\begin{tabular}{|llll|}
\hline Variable & $\begin{array}{l}\text { All patients } \\
(\mathrm{n}=100)\end{array}$ & $\begin{array}{l}\text { Elective } \\
(\mathrm{n}=50)\end{array}$ & $\begin{array}{l}\text { Fracture } \\
(\mathrm{n}=50)\end{array}$ \\
\hline Age & & & \\
\hline Min & $7 \mathrm{yrs}$ & $18 \mathrm{yrs}$ & $7 \mathrm{yrs}$ \\
\hline Max & $85 \mathrm{yrs}$ & $79 \mathrm{yrs}$ & $85 \mathrm{yrs}$ \\
\hline Mean & $48.7 \mathrm{yrs}$ & $55.2 \mathrm{yrs}$ & $43.9 \mathrm{yrs}$ \\
\hline Operative status & & \\
\hline Post op & 9 & 6 & 3 \\
\hline Pre op & 91 & 44 & 47 \\
\hline Orthopaedic issue & & \\
\hline Fracture & $50(50 \%)$ & 0 & $50(100 \%)$ \\
\hline Shoulder & $15(15 \%)$ & $15(30 \%)$ & 0 \\
\hline Knee & $14(14 \%)$ & $14(28 \%)$ & 0 \\
\hline Hip & $9(9 \%)$ & $9(18 \%)$ & 0 \\
\hline Elbow & $2(2 \%)$ & $2(4 \%)$ & 0 \\
\hline Foot or Ankle & $2(2 \%)$ & $2(4 \%)$ & 0 \\
\hline Other & $8(8 \%)$ & $8(16 \%)$ & 0 \\
\hline
\end{tabular}

Table 2:

Participant age summary

\begin{tabular}{|llll|}
\hline & $\begin{array}{l}\text { All patients } \\
(n=100)\end{array}$ & $\begin{array}{l}\text { Male } \\
(n=41)\end{array}$ & $\begin{array}{l}\text { Female } \\
(n=59)\end{array}$ \\
\hline$<25 \mathrm{yrs}$ & 21 & 12 & 9 \\
\hline $25-49 \mathrm{yrs}$ & 24 & 10 & 14 \\
\hline $50-74 \mathrm{yrs}$ & 42 & 16 & 26 \\
\hline$>75 \mathrm{yrs}$ & 13 & 3 & 10 \\
\hline
\end{tabular}


Table 3:

Participant one-way commute durations

\begin{tabular}{|llll|}
\hline & $\begin{array}{l}\text { All patients } \\
(n=100)\end{array}$ & $\begin{array}{l}\text { Elective } \\
(n=50)\end{array}$ & $\begin{array}{l}\text { Fracture } \\
(n=50)\end{array}$ \\
\hline Mean (mins) & 21 & 18 & 23 \\
\hline Std. dev & 13 & 11.7 & 13.9 \\
\hline Min (mins) & 2 & 5 & 2 \\
\hline Max (mins) & 60 & 60 & 60 \\
\hline
\end{tabular}

Table 4:

Questionnaire responses

\begin{tabular}{|c|c|c|c|c|}
\hline Questions & Mean & $\begin{array}{l}\text { Std. } \\
\text { dev. }\end{array}$ & Min & Max \\
\hline The telehealth appointment was convenient & 9.0 & 1.5 & 2 & 10 \\
\hline The instructions for appointment were clear & 9.2 & 1.5 & 1 & 10 \\
\hline I was satisfied with the wait time on the day of the appointment & 8.8 & 1.7 & 3 & 10 \\
\hline The doctor listening to me carefully & 9.1 & 1.3 & 3 & 10 \\
\hline The doctor explained things clearly and understandably & 9.0 & 1.7 & 1 & 10 \\
\hline I had enough time to communicate with the doctor & 9.1 & 1.5 & 2 & 10 \\
\hline $\begin{array}{l}\text { After the appointment, I understood the recommendation of the } \\
\text { doctor }\end{array}$ & 9.2 & 1.5 & 1 & 10 \\
\hline Overall, I was satisfied with the quality of the appointments & 9.2 & 1.7 & 3 & 10 \\
\hline
\end{tabular}


Table 5:

selection of patient responses to the qualitative component of the questionnaire

Positive

"good doctor, well explained, easier with phone consult"

"Very convenient, less waiting"

"impressed, no hassle, more informative than expected"

"Nice not to have to travel"

"Great service, good communication"

Negative

"The doctors expected me to know if something was wrong with my hip replacement"

"First appointments should be in person"

"seemed rushed"

"Frustrating if phone call is not on time"

"call not at the time stipulated"

\section{Figures}


1. Did you have to attend the hospital on the day of their phone appointment? (for $\mathrm{x}$-ray/cast application/cast removal etc)

Yes

2 No

2. Did you have to miss work/school or attend this appointment?

Yes

No

3. How convenient was having your outpatient clinic appoint via phone

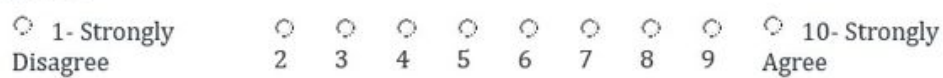

4. The instructions for the appointment were clear

$\begin{array}{lllllllllll}\text { 1-Strongly } & 2 & 2 & \ddots & 0 & 2 & 2 & 2 & 0 & 0 & 10 \text { - Strongly } \\ \text { Disagree } & 2 & 3 & 4 & 5 & 6 & 7 & 8 & 9 & \text { Agree }\end{array}$

5. I was satisfied with the wait time on the day of the appointment

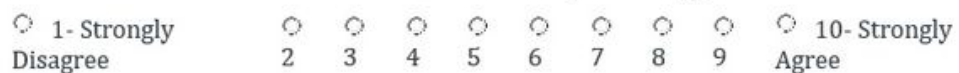

6. The doctor listened to me carefully

$\begin{array}{lllllllllll}2 \text { 1-Strongly } & 2 & 2 & 2 & 0 & 2 & 0 & 9 & 9 & 0 & 10 \text {-Strongly } \\ \text { Disagree } & 2 & 3 & 4 & 5 & 6 & 7 & 8 & 9 & \text { Agree }\end{array}$

7. The doctor explained things clearly and understandably

$$
\begin{aligned}
& \text { 1-Strongly } 20 \% \\
& \begin{array}{lllllllllll}
\text { Disagree } & 2 & 3 & 4 & 5 & 6 & 7 & 8 & 9 & \text { Agree }
\end{array}
\end{aligned}
$$

8. I had enough time to communicate with the doctor

$$
\begin{array}{lllllllllll}
2 & \text { 1-Strongly } & 2 & 2 & \ddots & 2 & 2 & 0 & 2 & 9 & 0 \\
\text { Disagree } & 2 & 3 & 4 & 5 & 6 & 7 & 8 & 9 & \text { Agree }
\end{array}
$$

9. After the appointment, I understood the recommendations of the doctor

$\begin{array}{lllllllllll}2 \text { 1-Strongly } & 2 & \ddots & \ddots & 2 & 2 & 2 & 2 & 9 & 0 & 10 \text { - Strongly } \\ \text { Disagree } & 2 & 3 & 4 & 5 & 6 & 7 & 8 & 9 & \text { Agree }\end{array}$

10. Overall I was satisfied with the quality of the appointments
1- Strongly

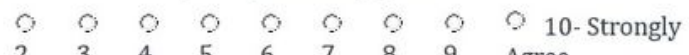
Disagree
$\begin{array}{lllllllll}2 & 3 & 4 & 5 & 6 & 7 & 8 & 9 & \text { Agree }\end{array}$

11. In future, would you prefer a face-to-face appointment or a phone appointment?

Face to Face

2 Phone

12. Do you have any Positive/Negative comments regarding your phone appointment?

\section{Figure 1}

Patient questionnaire

\section{Supplementary Files}


- PatientExperienceRAWDATA.xIsx

Page 15/15 